\title{
Hadronic Effects on Charmoium Elliptic Flows in Heavy-Ion Collisions
}

\author{
Baoyi Chen, ${ }^{1}$ Liu Jiang, ${ }^{1}$ and Yunpeng Liu ${ }^{1, *}$ \\ ${ }^{1}$ Department of Physics, Tianjin University, Tianjin 300350, China
}

(Dated: June 28, 2021)

\begin{abstract}
Transport and Langevin equations are employed to study hadronic medium effects on charmonium elliptic flows in heavy-ion collisions. In $\mathrm{Pb}-\mathrm{Pb}$ collisions, the anisotropic energy density of the quarkgluon plasma (QGP) in the transverse plane is transformed into hadron momentum anisotropy after the phase transition. Charmonia with high transverse momentum $p_{T}$ are produced via the primordial hard process and undergo different degrees of dissociation along different paths in the QGP. They then scatter with light hadrons in the hadron phase. Both contributions to the charmonium elliptic flows are studied at moderate and high transverse momenta. The elliptic flows of the prompt $J / \psi$ are found to be considerably enhanced at high transverse momentum when the charmonium diffusion coefficients in the hadronic medium are parametrized through the geometry scale approximation. This hadronic medium effect is negligible for quarkonia with larger mass such as bottomonia.
\end{abstract}

PACS numbers: 25.75.-q, 12.38.Mh, 14.40.Pq

\section{INTRODUCTION}

Charmonium consists of charm and anti-charm quarks. It is produced in parton hard scatterings with large momentum transfer. Charmonium has been considered as a clean probe of the hot dense medium created in relativistic heavy-ion collisions [1]. In the quark-gluon plasma (QGP), thermal light partons can dissociate charmonium states through inelastic collisions and the color screening effect, whereby the heavy quark potential in charmonium is screened by the light partons [2 7]. The charmonium dissociation rates induced by the two mechanisms are positively correlated to the QGP energy density [8]. At the Large Hadron Collider (LHC), QGP with large energy density dominates the abnormal suppression of charmonium yields compared with the effects of hadronic medium dissociations. Therefore, hadron phase contributions have been usually neglected in the analysis of the quarkonium nuclear modification factor $R_{A A}$ in the heavy-ion collisions at LHC collision energies. Furthermore, the hadronic medium produced after the violent expansion of QGP carries larger collective flows. This can affect the momentum distribution of charmonia through elastic scattering.

In nucleus-nucleus collisions, some charmonia are also produced through the combination of uncorrelated charm and anti-charm quarks in the QGP; this process is usually referred to as "regeneration" 9 12. With numerous (anti-)charm quarks moving randomly in the QGP, their combination probability at the hadronization surface becomes non-negligible. From both theoretical and experimental studies, it has been found that more than half of the final $J / \psi$ in $\mathrm{Pb}-\mathrm{Pb}$ central collisions are due to the combination of $c$ and $\bar{c}$ [13, 14. Being strongly coupled with the bulk medium, these charm quarks carry collective flows from the anisotropic expansion of the

\footnotetext{
*Electronic address: yunpeng.liu@tju.edu.cn
}

QGP [15, 16. This collective flow of charm quarks will be inherited by the regenerated charmonia [17. These regenerated charmonia are mainly located in the low $p_{T}$ region. In the high $p_{T}$ region where the regeneration contribution is negligible, charmonium yields are dominated by the primordial production. Primordially produced charmonia undergo different magnitudes of dissociations when they move in different directions in the anisotropic QGP [18. This effect results in an anisotropic charmonium momentum distribution after they have moved out of the QGP. This contributes $\sim 2 \%$ of the elliptic flow of $J / \psi$ in the high $p_{T}$ region in semi-central $\mathrm{Pb}$ $\mathrm{Pb}$ collisions, which is considerably lower than that obtained from experimental data 17. In this work, the hadronic medium effects on charmonium elliptic flows are studied, particularly in the high $p_{T}$ region. Charmonium trajectories are treated as Brownian motions in the hadronic medium, due to elastic scattering with thermal hadrons. This process can be described through the Langevin equation. The interactions between charmonium and the hadronic medium are parametrized via the diffusion coefficients in the Langevin equation [19].

The transport model is employed to study charmonium evolutions in the QGP [13, 20, while the Langevin equation is used to model charmonium diffusions in the hadronic medium. This paper is organized as follows. The transport and Langevin models are introduced in Section II. The numerical results and analysis are presented in Section III. Section IV provides a summary.

\section{TRANSPORT MODEL AND LANGEVIN MODEL}

The Boltzmann-type transport model has been developed to describe the phase space evolutions of heavy quarkonia in heavy-ion collisions. This model explains well and consistently most of the experimental data, including the spectrum and collective flows of charmonia and bottomonia [3, 20,23. The transport equation is 
written as:

$$
\partial_{t} f_{\psi}(t, \mathbf{x}, \mathbf{p})+\mathbf{v}_{\psi} \cdot \nabla_{\mathbf{x}} f_{\psi}(t, \mathbf{x}, \mathbf{p})=-\alpha f_{\psi}(t, \mathbf{x}, \mathbf{p})+\beta,
$$

where $f_{\psi}(t, \mathbf{x}, \mathbf{p})$ is the charmonium density in the phase space. The second term on the left-hand side represents the free streaming of charmonium with a constant velocity. In Eq.(1), the elastic collision term in the QGP [24] has been neglected. Hot medium effects on charmonia are parametrized through the $\alpha$ and $\beta$ functions. $\alpha$ is the charmonium dissociation rate in the QGP; it depends on the local temperatures and inelastic cross sections $(g+\psi \rightarrow c+\bar{c})$ between the charmonia and thermal partons. This cross section is obtained from the operator production expansion (OPE) [25, 26]. In the expression for the $\alpha$ dissociation rate, $J / \psi$ in-medium binding energy is taken to be $500 \mathrm{MeV}$. For the loosely bound excited states $\left(\chi_{c}, \psi^{\prime}\right)$, the dissociation cross sections calculated using the OPE are no longer reliable. For this reason, we choose in this work to obtain the dissociation rates of the excited charmonium states by phenomenologically parameterizing them in terms of a geometry scale with the ground state, as discussed below. The dissociation rates of the charmonium excited states are obtained through the geometry scale with the ground state. These parameters regarding $\alpha$ are the same as those reported in a previous work [13]. The $\beta$ term represents the charmonium regeneration rate due to the process of $c$ and $\bar{c}$ combination. As this contribution is negligible for the $J / \psi$ production in the high $p_{T}$ region, it is not included in the following calculations of $J / \psi$ elliptic flows.

The distributions of primordial charmonia in $\mathrm{Pb}-\mathrm{Pb}$ collisions are obtained through a superposition of effective nucleon-nucleon collisions, according to:

$$
\begin{aligned}
f_{t=0}\left(\mathbf{x}_{T}, \mathbf{p}_{T}, y \mid \mathbf{b}\right) & =T_{A}\left(\mathbf{x}_{T}+\frac{\mathbf{b}}{2}\right) T_{B}\left(\mathbf{x}_{T}-\frac{\mathbf{b}}{2}\right) \\
& \times \frac{d^{2} \sigma_{J / \psi}^{p p}}{d y 2 \pi p_{T} d p_{T}} \mathcal{R}_{s}\left(\mathbf{x}_{T}, \mathbf{p}_{T}, y\right),
\end{aligned}
$$

where $T_{A(B)}$ is the thickness function of the nucleus $\mathrm{A}(\mathrm{B}), \mathbf{b}$ is the impact parameter, $y$ is the rapidity, and $d^{2} \sigma_{J / \psi}^{p p} / d y 2 \pi p_{T} d p_{T}$ is the $J / \psi$ momentum distribution in pp collisions. $\mathcal{R}_{s}$ is the modification factor due to the shadowing effect; it is calculated via the EPS09 model [27]. At $\sqrt{s_{N N}}=5.02 \mathrm{TeV}$, the $J / \psi$ prompt differential cross section is taken to be $3.25 \mu b$ in the forward rapidity [28, 29].

In the hadronic medium, all the collisions between charmonia and light hadrons are parametrized in terms of the drag coefficient and noise term in the Langevin equation. The classical Langevin equation [30] for charmonium motion in a hadronic medium is written as:

$$
\frac{d \mathbf{p}}{d t}=-\eta \mathbf{p}+\xi
$$

where $\mathbf{p}$ is the charmonium momentum, while $\eta$ and $\xi$ are the drag force and the noise from the hadronic medium, respectively. For the simplicity of calculation, the classical form of the Langevin equation is assumed, and the momentum dependence of both $\eta$ and $\xi$ is neglected [31. $\xi$ satisfies the correlation relation:

$$
\left\langle\xi^{i}(t) \xi^{j}\left(t^{\prime}\right)\right\rangle=\kappa \delta^{i j} \delta\left(t-t^{\prime}\right),
$$

where $\kappa$ is the charmonium diffusion coefficient in the momentum space; it is connected with the spatial diffusion coefficient $\mathcal{D}_{s}^{\psi}$ through $\kappa=2 T^{2} / \mathcal{D}_{s}^{\psi}$ in the classical limit. The drag force in the Langevin equation can then be determined via the Einstein fluctuation-dissipation relation $\eta \approx \kappa / 2 m_{\psi} T$. Here, $m_{\psi}$ is the charmonium mass, and $T$ is the local temperature of the hadronic medium. In this equation, only one parameter, namely $\mathcal{D}_{s}^{\psi}$ for charmonium, is as yet undetermined. The diffusion coefficients of open heavy flavor mesons in the hadronic medium have been studied via the effective Lagrangian model and other models 32 35. The charmonium diffusion coefficients, in particular for the excited states, have not yet been determined. Lacking more rigorous calculations, we employ here the geometry scale approximation to extract the charmonium diffusion coefficients in the hadronic medium. Due to the uncertainty in the diffusion coefficients, different values are here considered to calculate the $J / \psi$ elliptic flows.

The $\mathrm{D}$ meson diffusion coefficient $\mathcal{D}_{s}^{D}$ in the hadronic medium has been calculated using the hadron resonance gas (HRG) model. It has been found that $4 \lesssim$ $\mathcal{D}_{s}^{D}(2 \pi T) \lesssim 10$ in the temperature region of $0.8 T_{c} \approx$ $T<T_{c}$ [36. The value of $\mathcal{D}_{s}^{D}(2 \pi T)$ increases when the temperature decreases in the hadron phase. In this work, we take $\mathcal{D}_{s}^{D}(2 \pi T)=8$ in the temperature region of $0.8 T_{c} \sim T_{c}$ for simplicity [36]. Additionally, the hadronic medium is assumed to reach kinetic freeze-out at $T_{\text {kin }}=0.8 T_{c}$. The charmonium diffusion coefficients are obtained via the geometry scale, $\mathcal{D}_{s}^{\psi}=\mathcal{D}_{s}^{D} \times \frac{\left\langle r_{D}\right\rangle^{2}}{\left\langle r_{\psi}\right\rangle^{2}}$. The charge radius of the $\mathrm{D}$ meson is approximated to be $\left\langle r_{D}\right\rangle=0.41 \mathrm{fm}$ 37. The radii of the charmonium ground state and excited states $\left(\chi_{c}, \psi^{\prime}\right)$ are 0.5 and $0.72 \mathrm{fm}$ [4, respectively. Based on this approximation, the charmonium excited states with relatively small spatial diffusion coefficients can develop large collective flows in the hadronic medium, which will increase the elliptic flows of the prompt and inclusive $J / \psi$ at high $p_{T}$ through the decay process $\chi_{c}\left(\psi^{\prime}\right) \rightarrow J / \psi X$.

Hot medium evolutions are simulated using the $(2+$ 1)-dimensional ideal hydrodynamic equations, and the longitudinal expansion is approximated with the Bjorken expansion:

$$
\partial_{\mu} T^{\mu \nu}=0
$$

where $T^{\mu \nu}=(e+p) u^{\mu} u^{\nu}-g^{\mu \nu} p$ is the energy-momentum tensor, while $e$ and $p$ are the energy density and pressure, respectively. Corrections due to viscous stresses in the hydrodynamic equations have been neglected here [38]. 
In the deconfined phase, the QGP is treated as an ideal gas of massless $u, d$ quarks and gluons, and $s$ quarks with $150 \mathrm{MeV}$ 39. The hadronic medium is treated as an ideal gas consisting of hadrons and resonances with mass up to $2 \mathrm{GeV}$ [3, 40]. The deconfined and confined phases are combined with a first-order phase transition at the critical temperature $T_{c}=165 \mathrm{MeV}$. At $\sqrt{s_{N N}}=5.02$ $\mathrm{TeV}$, the initial maximum temperatures of the QGP are fixed to be $450 \mathrm{MeV}$ in the forward rapidity $2.5<y<4$ in $\mathrm{Pb}-\mathrm{Pb}$ collisions [13.

\section{ELLIPTIC FLOWS OF CHARMONIUM}

Charmonium production is dominated by the regeneration mechanism at low $p_{T}$, while it is dominated by the primordial production at high $p_{T}$. The $J / \psi$ elliptic flows at low $p_{T}$ are mainly due to the kinetic thermalization of charm quarks and the regeneration process in the QGP. The ALICE collaboration presented a large elliptic flow of inclusive $J / \psi$ even at high $p_{T}$; which is higher than that of the theoretical calculations [17. The regeneration contribution is negligible at high $p_{T}$. One of the factors neglected in the theoretical studies is the charmonium diffusion in the hadronic medium. In this work, the hadron phase effects on $J / \psi$ collective flows are investigated using the Langevin equation.

The obtained charmonium elliptic flows are plotted in Fig 1. The dashed line includes contributions from the cold nuclear matter effects and QGP dissociations. The solid lines include contributions from cold nuclear matter effects, QGP dissociations, and diffusions in the hadronic medium. As we focus on the $J / \psi$ elliptic flows at high $p_{T}$, the regeneration contribution, which is mainly located in the $p_{T} \lesssim 4 \mathrm{GeV} / \mathrm{c}$ region, is deliberately neglected in these calculations. Based on the geometry scale approximation, different charmonium states $\left(J / \psi, \chi_{c}, \psi^{\prime}\right)$ take different values of the diffusion coefficients in the hadronic medium. The charmonium excited states can therefore obtain larger collective flows from the expanding hadronic medium due to a smaller diffusion coefficient. This will increase the $v_{2}$ of the inclusive $J / \psi$ through the $\chi_{c}\left(\psi^{\prime}\right) \rightarrow J / \psi X$ decay process after the evolutions in the QGP and hadron phase, as shown by the black solid line in Fig.1. Due to the uncertainty in the charmonium diffusion coefficients, different values are also considered (refer to the red and blue lines in Fig 11). In the case of the red line, all charmonium states take the same value, i.e., $\mathcal{D}_{s}^{\psi}(2 \pi T)=8$. The hadronic medium effects become smaller. In the case of the blue line, all charmonium states take a different value, namely $\mathcal{D}^{\psi}(2 \pi T)=15$. Both these situations yield smaller elliptic flows of the inclusive $J / \psi$, as the diffusion coefficients in these two scenarios are larger than the value obtained from the geometry scale approximation.

In the $40-60 \%$ centrality, some of the charmonium excited states can survive the QGP dissociations and carry elliptic flows from the hadronic medium if their spa-

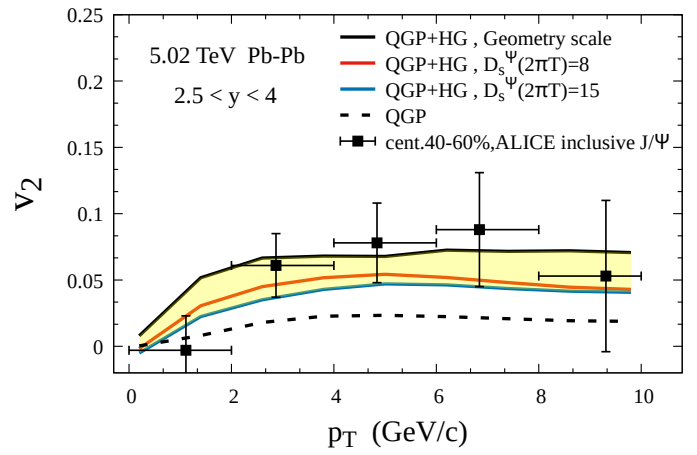

FIG. 1: (Color online) Elliptic flows of the primordial $J / \psi$ as a function of the transverse momentum in cent.40-60\% in $\sqrt{s_{N N}}=5.02 \mathrm{TeV} \mathrm{Pb}-\mathrm{Pb}$ collisions. The dashed line represents $v_{2}$ of the primordial $J / \psi$ with QGP dissociations. The solid lines include both the QGP dissociations and diffusions in the hadronic medium. The diffusion coefficients determined by the geometry scale, $\mathcal{D}_{s}^{\psi}(2 \pi T)=8$, and $\mathcal{D}_{s}^{\psi}(2 \pi T)=15$, are plotted with black, red, and blue solid lines, respectively. The decay contributions $\left(\chi_{c} \rightarrow J / \psi X, \psi^{\prime} \rightarrow J / \psi X\right)$ are included in all lines. The regeneration and B-hadron decay parts are not included in the theoretical lines. The experimental data represents the inclusive $J / \psi$ from the ALICE collaboration [17].

tial diffusion coefficients $\mathcal{D}_{s}^{\psi}$ are small. Considering that hadron elastic scatterings can increase the $v_{2}$ of B mesons by $\sim 2 \%$ [15, 16, a larger effect in the case of charmonium excited states is expected since their masses are smaller and the geometry size is larger. As Fig 1 shows, the hadronic effects result in an increase of $v_{2}^{J / \psi}$ from $\sim 2 \%$ to $\sim 6 \%$ at $p_{T} \sim 6 \mathrm{GeV} / \mathrm{c}$ when the charmonium diffusion coefficients are extracted using the geometry scale approximation. It should be noted that at low $p_{T}$, after including the regeneration contribution, the inclusive $J / \psi v_{2}$ is still dominated by the regeneration process.

Fig 2 shows the calculations in cent.20-40\%. The hadronic effects result in an increase in the elliptic flow of the primordial $J / \psi$ from $\sim 2.5 \%$ to $\sim 5 \%$. The effect is slightly smaller than that observed in cent.40-60\%. This is because a larger proportion of the charmonium excited states is dissociated in the QGP, and their contribution to the final $v_{2}^{J / \psi}$ is suppressed. In more central collisions such as cent.5-20\%, the hadronic effects become smaller than those illustrated in Figs 1. 2, The addition of the hadron phase leads to an enhancement of $v_{2}$ of the primordial $J / \psi$; furthermore, this addition reduces the nuclear modification factor $R_{A A}$ of $J / \psi$ at $p_{T} \gtrsim 5 \mathrm{GeV} / \mathrm{c}$ where the primordial production dominates, as shown in Fig, 3 .

Fig 4 shows the hadronic effects on $\Upsilon(1 \mathrm{~S}$ ) (red solid line). As bottomonia are tightly bound states, the QGP dissociations contribute only $\sim 1 \%$ to the $v_{2}$ of $\Upsilon(1 \mathrm{~S})$ through path length difference effect [18. The difference between the red solid line and the red dashed line in 


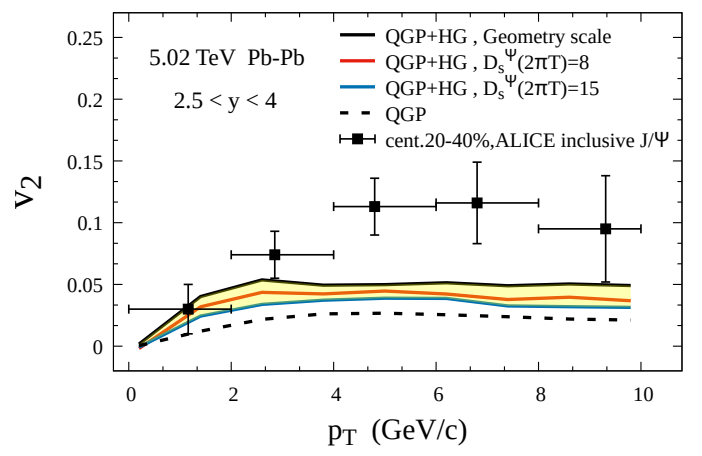

FIG. 2: (Color online) Elliptic flows of the primordial $\mathrm{J} / \psi$ as a function of the transverse momentum in cent.20-40\% in $\sqrt{s_{N N}}=5.02 \mathrm{TeV} \mathrm{Pb}-\mathrm{Pb}$ collisions. The dashed line represents $v_{2}$ of the primordial $J / \psi$ with QGP dissociations. Solid lines include both QGP and hadronic medium effects. The other parameters are the same as in Fig 1. The experimental data represents the inclusive $J / \psi$ obtained from the ALICE collaboration 17.

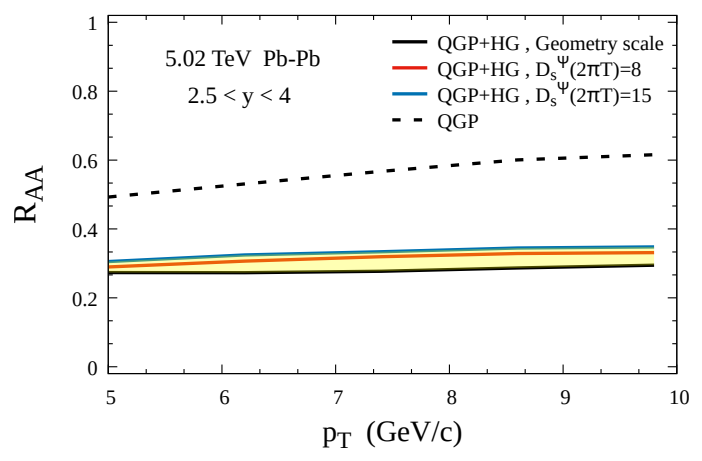

FIG. 3: (Color online) $J / \psi$ nuclear modification factor $R_{A A}$ as a function of $p_{T}$ in cent.40-60\%. The other parameters are the same as in Fig 1 The dashed line is obtained considering QGP effects only, while the black, red, and blue solid lines correspond to the situations where the charmonium diffusion coefficients are determined by the geometry scale, $\mathcal{D}_{s}^{\psi}(2 \pi T)=$ 8 , and $\mathcal{D}_{s}^{\psi}(2 \pi T)=15$, respectively.

Fig 4 represents the effect of the hadronic medium on the $v_{2}$ of $\Upsilon(1 \mathrm{~S})$; this effect is negligible due to the upsilon large mass and small geometry size. In addition, the substantial difference between the elliptic flows of $\mathrm{J} / \psi$ and $\Upsilon(1 S)$ in Fig 4 indicates that the former come from the final state interactions rather than from the initial cold nuclear matter effects [42]. This is beneficial to the understanding of the origin of the charmonium collective behaviors in the hot medium.

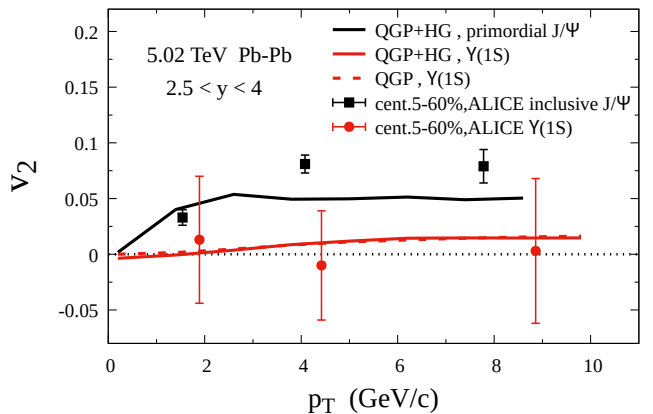

FIG. 4: (Color online) Elliptic flows of the inclusive $J / \psi$ and $\Upsilon(1 \mathrm{~S})$ as a function of $p_{T}$ in the forward rapidity $2.5<y<4$ in cent.5-60\% in $\sqrt{s_{N N}}=5.02 \mathrm{TeV} \mathrm{Pb-Pb}$ collisions. The theoretical calculations of $J / \psi$ are the same as those presented in Fig. The diffusion coefficients of $J / \psi$ and $\Upsilon(1 S)$ are obtained using the geometry scale. Regeneration is absent here. The experimental data are obtained from the ALICE collaboration [4].

\section{SUMMARY}

The transport and Langevin equations were employed to study the elliptic flows of primordial charmonium in the QGP and hadron phase. Charmonium diffusions in the hadronic medium were simulated using the Langevin equation. When the spatial diffusion coefficients in the Langevin equation are parametrized via the geometry scale approximation, the charmonium $v_{2}$ can be enhanced by $\sim 5 \%$ at high $p_{T}$ after considering the hadronic medium effects. This contribution would be suppressed if a larger value of the diffusion coefficient was considered. The $J / \psi$ nuclear modification factor at high $p_{T}$ is also suppressed by the diffusion process. The bottomonium $v_{2}$ is not substantially affected by these hadronic medium effects due to the large bottomonium mass. The difference between the charmonium and bottomonium experimental data indicates that the elliptic flows of charmonium at high $p_{T}$ come from the final state interactions in the hot medium. The study of charmonium diffusions in the hadronic medium aids the understanding of the experimental data of quarkonium collective flows in ultrarelativistic heavy ion collisions.

Acknowledgements: $\mathrm{BC}$ is grateful to Wenbin Zhao for discussions regarding the hydrodynamic model. This work is supported by the NSFC Grant No. 11705125 and the "Qing-Gu" project (2019XRG-0066) of Tianjin University. 
(2001)

[3] X. l. Zhu, P. f. Zhuang and N. Xu, Phys. Lett. B 607, 107 (2005)

[4] H. Satz, J. Phys. G 32, R25 (2006)

[5] J. P. Blaizot, D. De Boni, P. Faccioli and G. Garberoglio, Nucl. Phys. A 946, 49 (2016)

[6] X. Yao and B. Muller, Phys. Rev. D 100, no. 1, 014008 (2019)

[7] X. Yao and T. Mehen, Phys. Rev. D 99, no. 9, 096028 (2019)

[8] B. Chen, M. Hu, H. Zhang and J. Zhao, Phys. Lett. B 802, $135271(2020)$

[9] R. L. Thews, M. Schroedter and J. Rafelski, Phys. Rev. C 63, 054905 (2001)

[10] A. Andronic, P. Braun-Munzinger, K. Redlich and J. Stachel, Phys. Lett. B 571, 36 (2003)

[11] L. Yan, P. Zhuang and N. Xu, Phys. Rev. Lett. 97, 232301 (2006)

[12] X. Du and R. Rapp, Nucl. Phys. A 943, 147 (2015)

[13] B. Chen, Chin. Phys. C 43, no. 12, 124101 (2019)

[14] X. Zhao and R. Rapp, Nucl. Phys. A 859, 114 (2011)

[15] M. He, R. J. Fries and R. Rapp, Phys. Lett. B 735, 445 (2014)

[16] S. Cao, G. Y. Qin and S. A. Bass, Phys. Rev. C 92, no. 2, 024907 (2015)

[17] S. Acharya et al. [ALICE Collaboration], Phys. Rev. Lett. 119, no. 24, 242301 (2017)

[18] P. P. Bhaduri, N. Borghini, A. Jaiswal and M. Strickland, Phys. Rev. C 100, no. 5, 051901(R) (2019)

[19] S. Mitra, S. Ghosh, S. K. Das, S. Sarkar and J. e. Alam, Nucl. Phys. A 951, 75 (2016)

[20] K. Zhou, N. Xu, Z. Xu and P. Zhuang, Phys. Rev. C 89, no.5, $054911(2014)$

[21] B. Chen, T. Guo, Y. Liu and P. Zhuang, Phys. Lett. B 765, $323(2017)$

[22] Y. Liu, B. Chen, N. Xu and P. Zhuang, Phys. Lett. B 697, 32 (2011)

[23] X. Yao, W. Ke, Y. Xu, S. A. Bass and B. Müller, arXiv:2004.06746 [hep-ph].
[24] B. Chen, K. Zhou and P. Zhuang, Phys. Rev. C 86, 034906 (2012)

[25] M. E. Peskin, Nucl. Phys. B 156, 365 (1979).

[26] G. Bhanot and M. E. Peskin, Nucl. Phys. B 156, 391 (1979).

[27] K. J. Eskola, H. Paukkunen and C. A. Salgado, JHEP 0904, 065 (2009)

[28] B. Abelev et al. [ALICE Collaboration], Phys. Lett. B 718, 295 (2012) Erratum: [Phys. Lett. B 748, 472 (2015)]

[29] B. Abelev et al. [ALICE Collaboration], JHEP 1211, 065 (2012)

[30] B. Chen and J. Zhao, Phys. Lett. B 772, 819 (2017)

[31] R. Rapp and H. van Hees, Quark-Gluon Plasma 4, pp. 111-206 (2010), arXiv:0903.1096 [hep-ph].

[32] S. K. Das, S. Ghosh, S. Sarkar and J. e. Alam, Phys. Rev. D 85, 074017 (2012)

[33] L. M. Abreu, D. Cabrera, F. J. Llanes-Estrada and J. M. Torres-Rincon, Annals Phys. 326, 2737 (2011)

[34] L. M. Abreu, D. Cabrera and J. M. Torres-Rincon, Phys. Rev. D 87, no. 3, 034019 (2013)

[35] L. Tolos and J. M. Torres-Rincon, Phys. Rev. D 88, 074019 (2013)

[36] M. He, R. J. Fries and R. Rapp, Phys. Rev. Lett. 110, no. 11,112301 (2013)

[37] J. Zhao, K. Zhou, S. Chen and P. Zhuang, arXiv:2005.08277 [nucl-th].

[38] C. Shen, Z. Qiu, H. Song, J. Bernhard, S. Bass and U. Heinz, Comput. Phys. Commun. 199, 61-85 (2016).

[39] J. Sollfrank, P. Huovinen, M. Kataja, P. V. Ruuskanen, M. Prakash and R. Venugopalan, Phys. Rev. C 55, 392 (1997)

[40] K. Hagiwara et al. (Particle Data Group), Phys. Rev. D 66, 010001(R) (2002).

[41] S. Acharya et al. [ALICE Collaboration], Phys. Rev. Lett. 123, no. 19, 192301 (2019)

[42] C. Zhang, C. Marquet, G. Y. Qin, S. Y. Wei and B. W. Xiao, Phys. Rev. Lett. 122, no. 17, 172302 (2019) 